\title{
¿SON LAS COOPERATIVAS MÁS FAVORABLES A LA \\ PRESENCIA DE MUJERES EN LOS CONSEJOS QUE OTRAS ENTIDADES? ${ }^{1}$
}

\author{
POR \\ María Luisa ESTEBAN SALVADOR ${ }^{2}$
}

\section{RESUMEN}

Este trabajo analiza en qué empresas se cuenta con órganos de gobierno más favorables a la participación femenina en el periodo previo al desarrollo de medidas y recomendaciones sobre la presencia de mujeres en los consejos de administración de las grandes sociedades españolas, entre las que se incluyen cooperativas. Asimismo pone en relación la rentabilidad con el género en sus órganos de gobierno. Para ello, en un primer análisis se estudia la rentabilidad económica de las sociedades no financieras cotizadas en el mercado continuo y de aquellas que aunque no cotizan en dicho mercado están obligadas a presentar un Informe Anual de Gobierno Corporativo a la Comisión Nacional del Mercado de Valores desde el año 2004. Posteriormente se reduce la muestra tras la exclusión de las empresas no cotizadas en el mercado continuo. Los resultados muestran que una única entidad contribuyó a cambiar la rentabilidad y se coloca como modelo de estudio. Una característica diferenciadora de esta empresa es su forma jurídica, ya que se trata de una empresa cooperativa.

\footnotetext{
${ }^{1}$ La autora quiere agradecer a los revisores anónimos sus comentarios a la versión previa de este artículo, y al proyecto TECO2008-03179-E/ECON del Ministerio de Ciencia e Innovación (MICINN), su financiación.

${ }^{2}$ Profesora titular de la Universidad de Zaragoza en la Facultad de Ciencias Sociales y Humanas, Departamento de Contabilidad y Finanzas. Ciudad Escolar s/n, 44003 Teruel (Spain). Dirección de correo electrónico: luisaes@unizar.es.
}

REVESCO No 110 - Primer Cuatrimestre 2013 - ISSN: 1885-8031 - www.ucm.es/info/revesco

http://dx.doi.org/10.5209/rev_REVE.2013.v110.41443

Fecha de recepción: 22/08/2011

Fecha de aceptación: 13/04/2012 
Palabras clave: Género; gobierno corporativo, economía social, paridad, consejo rector.

Claves Econlit: G300, G390, J160, P130

\title{
ARE THE COOPERATIVES IN MORE FAVOURABLE OF HAVING WOMEN IN THEIR BOARD OF DIRECTORS THAN OTHER ENTITIES?
}

\begin{abstract}
This paper analyzes which companies have more women on their board of directors in the moment previous to the publication of measures and recommendations about the female presence on the boards of directors of the Spanish big companies, including cooperatives. At the same time the study puts in relation the gender on boards with the return on assets. First, the current work starts with the sample of non-financial firms obliged to present an Annual Corporate Governance Report to the Spanish Securities Market Commission with data from 2004. Second, the sample is reduced; excluding the companies not listed in the continuous market. The results indicate that only one company contributed to change the return on assets and becomes a model to study. One characteristic of this entity is their juridical form, as a cooperative.
\end{abstract}

Keywords: Gender; corporate governance, social economy, parity, director.

\section{INTRODUCCIÓN}

En las llamadas sociedades de participación democrática la implicación directa de las mujeres en la gestión es mayor que en otro tipo de entidades (Mateos, Escot y Gimeno, 2006; Mateos, Iturroiz, y Gimeno, 2009; Mateos, Gimeno y Escot, 2010). Mateos, Iturroiz, y Gimeno (2009) evidencian que los principios inspiradores de las cooperativas y las peculiaridades de estas entidades posibilitan el ascenso de las mujeres dentro de la organización jerárquica. De hecho, algunos autores encuentran en estas entidades un instrumento eficaz para conseguir las grandes metas humanitarias, entre las que se encuentra el papel de la mujer (García, 2010). Con todo, distintos trabajos muestran que la presencia femenina en los órganos de gobierno de las entidades enmarcadas en la Economía Social es 
inferior a la masculina (Chaves, Ribas y Sajardo, 2005; Esteban, Gargallo y Pérez, 2010; Ribas, 2006; Sajardo, Ribas, Benavent, Saz, Serra y Vilar, 2009).

El papel de las mujeres en las cooperativas se muestra como una "foto borrosa y desenfocada" (Senent, 2011, 80) debido a la insuficiencia, dispersión y escasa actualización de datos diferenciados por género. De acuerdo con Senent (2011) las mujeres en el sector cooperativo son tratadas de manera desigual respecto a sus homólogos masculinos aun teniendo en cuenta la tradición y sensibilidad del sector cooperativo a tratamientos discriminatorios de género, y todo ello es debido a la influencia de contexto de la realidad social en el que estas entidades se encuentran inmersas ${ }^{3}$. En el trabajo realizado por Orellana y Celis (2011) se observa que la naturaleza de la actividad desarrollada por las mujeres directivas y su visión del trabajo de gestión responde a un estilo de dirección contemporáneo y tienen un mejor encaje en el entorno de las cooperativas de trabajo asociado identificado por las relaciones entre iguales.

Los estudios realizados muestran una desigual participación de las mujeres directivas en el perfil de estas entidades (Berenguer, Cerver, de la Torre y Torcal, 2004). Martínez, Arcas y García (2011) observan que mientras que las cooperativas y sociedades laborales cumplen con la paridad mínima establecida por la Ley Orgánica 3/2007 respecto al número de mujeres trabajadoras, entorno al $40 \%$, no ocurre lo mismo en puestos de toma de decisiones donde este porcentaje es mucho más bajo ${ }^{4}$, algunos estudios estiman que la participación de las mujeres en la gerencia de las entidades de la economía social es del 28,1\% (Sajardo et al., 2009). En el trabajo llevado a cabo por la Federación Valenciana de Cooperativas de Trabajo Asociado (FEVECTA, 1999) se concluye que el 37'7\% de los cargos directivos en las cooperativas analizadas están ocupados por mujeres, concretamente representan el $355^{\prime} 51 \%$ de puestos de los comités de dirección y el 27’47\% de los de los Consejos Rectores.

Un mayor número de mujeres en los órganos representativos de las cooperativas puede ayudar a que en las reuniones se logre que las condiciones de trabajo mejoren y se contribuya a mediar para conseguir un equilibrio económico, social y cultural en este tipo de entidades (Fregidou-Malama, 2004). Elío (2007), analiza la participación de las mujeres en los Consejos Rectores de las cooperativas de Mondragón Corporación Cooperativa encontrando diferencias

\footnotetext{
${ }^{3}$ Un interesante estudio de las teorías que analizan las desigualdades de género en el mercado de trabajo se puede encontrar en el trabajo desarrollado por Ribas y Sajardo (2005).

${ }^{4}$ Sobre el debate relativo al potencial democrático de las recientes intervenciones políticas respecto a la composición de los consejos véase Mcphail (2010).
} 
entre géneros en aspectos relativos a la remuneración percibida así como en los cargos ocupados. Mateos, Gimeno y Escot (2010) evidencian que es en las empresas familiares, en las cooperativas, y en general, en aquellas en las que los accionistas tienen un mayor poder a la hora de nombrar consejeros donde se hallan las proporciones más altas de consejeras. En Suecia, la participación femenina en los consejos de administración de las empresas privadas es más elevada que en otros países europeos, aunque de acuerdo con Fregidou-Malama (2004) es baja, sin embargo, la presencia de mujeres en los consejos de las cooperativas de consumo según un estudio llevado a cabo por el Instituto de Cooperativas Suecas es del $41 \%$ (Fregidou-Malama, 2004).

Algunas investigaciones muestran fuertes lazos entre la participación de las mujeres en los consejos, la rentabilidad y el buen gobierno corporativo (Bonn, 2004; Carter, Simkins y Simpson, 2003; Erhardt, Werbel y Shrader, 2003; The Boston Club, 2004). Carter et al. (2003) analizan si la diversidad en los consejos tiene que ver con la mejora en el valor financiero. Los mencionados autores evidencian una relación positiva entre la fracción de mujeres o minorías en el consejo y el valor de la empresa. En un trabajo posterior Carter, D’Souza, Simkins y Simpson (2010) encuentran que las mujeres y minorías étnicas deberían incorporarse a los consejos por criterios distintos a los rendimientos financieros futuros de las empresas.

Diferentes autores han cuestionado si es la presencia femenina en un consejo lo que mejora el rendimiento de la firma o si son las empresas más rentables más propensas a incorporar a mujeres en sus consejos (Brown, Brown y Anastasopoulos, 2002; Farrell y Hersch, 2005; Smith, Smith y Verner, 2006). Smith et al. (2006) encontraron que la relación positiva fue debida a la primera cuestión, es decir que la diversidad afecta al rendimiento, y no lo contrario. A pesar de que las entidades con mejores resultados tendieron a contar con más mujeres en los órganos de gobierno, no pudieron concluir que consejos más diversos en género generasen mejores resultados a la firma (Farrell y Hersch, 2005). Algunos trabajos asocian la diversidad con rendimientos negativos (Shrader Blackburn e Iles, 1997; Pelled, Eisenhardt y Xing, 1999; Bøhren y Strøm, 2006). En otro orden de cosas, García Lara, García Osma y Mora (2007) analizan si la diversidad de género en los consejos contribuye a mejorar la calidad de las cifras contables. Los mencionados autores encuentran una positiva pero débil asociación entre el porcentaje de mujeres consejeras y varias medidas de calidad de los beneficios. 
En el caso español, Campbell y Mínguez Vera (2008) encuentran para una muestra de 68 empresas no financieras cotizadas en el mercado continuo durante el periodo 1995 a 2000 que la diversidad del consejo medida por el porcentaje de mujeres tiene un impacto positivo en el valor de la firma. Para ello utilizan el indicador de mercado $Q$ de Tobin como una medida del valor de la empresa y lo consideran mejor que las medidas contables porque refleja las expectaciones de futuras ganancias en el mercado. Mateos, Escot y Gimeno (2006) no observaron diferencias estadísticamente significativas entre la presencia femenina en los consejos y la rentabilidad medida a través del ROA y de la productividad por empleado. En la esfera regional Redondo y Jimeno (2005) analizaron las relaciones económico financieras de los miembros de los consejos de administración de una muestra de de 2.009 empresas de Castilla-León de distintos tamaños y sectores con ingresos de explotación superiores a los 2.900.000 euros en 2002. En la mencionada investigación no se detectaron diferencias significativas ni para la rentabilidad económica ni para la financiera respecto a que el órgano de administración contase con más hombres, mujeres o existiese paridad.

\section{OBJETIVOS DE LA INVESTIGACIÓN, METODOLOGÍA E HIPÓTESIS}

El objetivo de este trabajo es, en primer lugar, analizar si las grandes sociedades cooperativas españolas cuentan con órganos de gobierno más favorables a la participación femenina que otro tipo de entidades respecto a su forma jurídica en un periodo previo a la proliferación de normativa e indicaciones sobre la presencia de mujeres en los consejos de administración. Entre las iniciativas llevadas a cabo en España cabe señalar el Informe del grupo especial de trabajo sobre buen gobierno de las sociedades cotizadas, aprobado el 19 de mayo de 2006, en el que se indica la recomendación de potenciar la participación de las mujeres en los órganos de administración. Un año después de ver la luz el mencionado código de buen gobierno, la Ley Orgánica 3/2007, de 22 de marzo para la igualdad efectiva de mujeres y hombres sugiere una presencia equilibrada de mujeres y hombres en los consejos en un plazo de ocho años a partir de su entrada en vigor. En este estudio se trata de evidenciar si en una etapa en que la presencia femenina en los consejos estaba menos expuesta a su observación, había entidades que de forma "natural" eran más propensas a la participación de las mujeres en sus máximos órganos de gobierno. La fecha del estudio es importante porque en esos momentos no había recomendaciones en España sobre la presencia femenina en los consejos, por lo que el hecho de que existiesen mujeres en determinados consejos no estaba asociado a las sugerencias que años posteriores fueron impulsadas. 
En segundo lugar, esta investigación trata de relacionar la rentabilidad económica con dos indicadores de género, en los consejos de administración en el caso de sociedades anónimas o en los rectores en el caso de cooperativas, de las grandes empresas españolas: el número de mujeres y el porcentaje de mujeres en cada consejo, con el fin de analizar si existen empresas que destacan por ser más rentables a la vez que presentar un mayor número de mujeres en sus órganos de gobierno. Para la realización de los análisis de rentabilidad se toman dos muestras con datos correspondientes a 31 de diciembre de 2004, mientras que para los análisis previos se utilizan datos de 2003 y 2004. Estos años son empleados en el estudio porque por primera vez en 2003 determinados tipos de sociedades están obligadas a presentar un Informe Anual sobre Gobierno Corporativo a la Comisión Nacional del Mercado de Valores, y entre la información obligada a publicar destaca la composición detallada de los órganos de gobierno.

En la Tabla 1 se presenta la distribución de mujeres en los consejos de los dos grupos de entidades analizados. Las cifras más favorables a las mujeres se alcanzaron el año 2004 en el grupo de entidades no cotizadas, donde el porcentaje de empresas sin mujeres en sus consejos fue del $33 \%$. En ese ejercicio la cifra de consejos sin presencia femenina fue casi el doble para el caso de las entidades cotizadas, alcanzando el 65\%. También fue en el mercado no continuo donde se logró el mayor número de asientos por consejo ocupados por mujeres. En 2004 en un $2 \%$ de las empresas siete mujeres coincidieron en un mismo consejo. 
Tabla 1. Número de mujeres en los consejos

\begin{tabular}{|c|c|}
\hline $\begin{array}{l}\text { De empresas que no cotizan en el } \\
\text { Mercado Continuo }\end{array}$ & $\begin{array}{c}\text { De empresas que cotizan en el } \\
\text { Mercado Continuo }\end{array}$ \\
\hline 2004 & 2003 \\
\hline
\end{tabular}

$\begin{array}{lllllllll}\text { Número de } & \mathbf{N}^{\circ} & \% & \mathbf{N}^{\circ} & \% & \mathbf{N}^{\circ} & \% & \mathbf{N}^{\circ} & \%\end{array}$ consejos con:

\begin{tabular}{ccccccccc}
\hline 0 mujeres & 30 & $45 \%$ & 38 & $33 \%$ & 81 & $66 \%$ & 80 & $65 \%$ \\
\hline 1 mujer & 19 & $29 \%$ & 29 & $25 \%$ & 30 & $24 \%$ & 34 & $28 \%$ \\
\hline 2 mujeres & 14 & $21 \%$ & 26 & $22 \%$ & 7 & $6 \%$ & 5 & $4 \%$ \\
\hline 3 mujeres & 1 & $2 \%$ & 8 & $7 \%$ & 3 & $2 \%$ & 2 & $2 \%$ \\
\hline 4 mujeres & 1 & $2 \%$ & 6 & $5 \%$ & 1 & $1 \%$ & 2 & $2 \%$ \\
\hline 5 mujeres & 0 & $0 \%$ & 7 & $6 \%$ & 0 & $1 \%$ & 0 & $0 \%$ \\
\hline 7 mujeres & 0 & $0 \%$ & 2 & $2 \%$ & 0 & $0 \%$ & 0 & $0 \%$ \\
\hline
\end{tabular}

Fuente: Elaboración propia

Para la consecución del segundo objetivo, y con el fin de poder realizar comparaciones homogéneas, se excluyen a las entidades de los sectores financiero y de seguros. De este modo, la primera muestra formada por aquellas entidades obligadas a presentar un Informe Anual de Gobierno Corporativo a la Comisión Nacional del Mercado de Valores cuenta con 
un total de 154 empresas, mientras que la segunda, compuesta únicamente por entidades cotizadas en el mercado continuo incluye a 104 entidades.

Como variable dependiente para evaluar la creación de valor se utiliza la medida contable de rendimiento rentabilidad económica. Para relacionar la rentabilidad económica con la variable independiente número de mujeres primero y con la variable porcentaje de mujeres después, se aplica la prueba Anova de un factor. Dado el carácter cuantitativo de las variables independientes, se tratan como ordinales. Con respecto a la variable número de mujeres, se toma como cualitativa. Por lo que se refiere a la variable porcentaje de mujeres, se define como ordinal una vez codificada. De este modo, tras haber convertido las variables métricas número y porcentaje de mujeres en variables ordinales, se aplican las pruebas de diferencias de medias. Se contrastan las hipótesis de que las medias poblacionales de la variable dependiente en cada nivel de las variables independientes son similares. En un segundo análisis, tras eliminar a las sociedades no cotizadas en el mercado continuo, se realizan las mismas exploraciones.

\section{RESULTADOS DE LOS ANÁLISIS}

\subsection{Los consejos más favorables a la participación femenina}

Con los datos del ejercicio 2003, se advierte que solamente en dos consejos el número de mujeres superó al de hombres, se trató de empresas no cotizadas en el mercado continuo y con un tamaño de consejo muy pequeño. En el primer caso INMUEBLES DE CAJA HIPOTECARIA CATAlANA MUTUAL, S. A. (PLARREGA INVEST, 2000, S. A.) que contó con un consejo unipersonal y en el segundo CALPE INVEST, S. A. con un consejo mayoritariamente femenino (de sus únicos cinco miembros, tres fueron mujeres, y una de ellas presidenta). En otros cinco consejos hubo una participación paritaria entre hombres y mujeres. Excepto en una de las entidades, EROSKI SOCIEDAD COOPERATIVA, donde el tamaño del consejo fue mayor, el resto fueron consejos muy pequeños, de 4 miembros, y la mayoría de estos consejos paritarios estuvieron presididos por mujeres. También cabe destacar que las siete entidades en las que el número de mujeres consejeras superó o igualó al de hombres no cotizaron en el mercado continuo, y que cinco de éstas sociedades estuvieron presididas por mujeres.

En cuanto al ejercicio 2004 los datos muestran que las siete primeras compañías con mayor participación femenina no cotizaron en el mercado continuo. En tres de éstas las 
mujeres superaron a los hombres $\mathrm{y}$, en dos, hubo consejos paritarios. A excepción de la sociedad con consejo unipersonal INMUEBLES DE CAJA HIPOTECARIA CATALANA MUTUAL, S. A. representada por una mujer, otra entidad CALPE INVEST, S. A., además de contar con un $80 \%$ de mujeres estuvo presidida por una de ellas, y una tercera, la SOCIEDAD COOPERATIVA EROSKI contó con más consejeras que consejeros. En dos entidades se observó un número paritario de miembros de ambos sexos en sus consejos, en INDUSTRIAS DEL CURTIDO, S. A. y en S. A. RONSA.

Ni un solo consejo de las entidades pertenecientes al mercado continuo contó con más mujeres que hombres. Es más, ni siquiera se contó con consejos paritarios en este grupo de entidades por lo que se puede concluir que, en general, las empresas más influyentes de nuestro país fueron menos propicias a incorporar a mujeres que otras menos prestigiosas.

El porcentaje más alto de mujeres en los consejos de las entidades cotizadas en el mercado continuo fue el obtenido por la empresa TRANSPORTES AZKAR, S. A. con un $40 \%$ de participación femenina, seguido del alcanzado por PROSEGUR COMPAÑIA DE SEGURIDAD, S. A. con un 33,33\% de mujeres, y presidido por una de ellas. Cabe señalar que la entidad TRANSPORTES AZKAR, S. A. además de incluir a cuatro mujeres consejeras contó con una mujer secretaria sin la condición de consejera.

\subsubsection{Valores extremos: número de mujeres}

En la Tabla 2 se muestran los valores extremos de las entidades con mayor y menor número de mujeres en sus consejos, diferenciadas por tipo de mercado. Ambos ejercicios y en los dos tipos de mercados, las empresas con consejos más favorables a las mujeres radicaron en el País Vasco. Así, las dos entidades en las primeras posiciones en el ranking respecto al número de mujeres el año 2003 con sede social en el País Vasco fueron: EROSKI SOCIEDAD COOPERATIVA y FAGOR ELECTRODOMÉSTICOS, SOCIEDAD COOPERATIVA con seis y cuatro mujeres en sus consejos respectivamente. Además, cabe señalar que la forma jurídica de ambas entidades se diferencia de la mayoría de las empresas de la muestra por tratarse de sociedades cooperativas. En el grupo de entidades cotizadas en el mercado continuo, también una empresa vasca TRANSPORTES AZKAR, S. A., ocupó el primer ranking, posición que seguiría ocupando en 2004, con cuatro mujeres en su consejo los dos periodos analizados. 
El ejercicio 2004 EROSKI SOCIEDAD COOPERATIVA y CAJA INSULAR DE AHORROS DE CANARIAS fueron las entidades mejor posicionadas en cuanto a número de mujeres, empatadas, con siete mujeres en su consejo cada una. Se aprecian diferencias entre los ejercicios 2003 y 2004 debidas a la incorporación de las Cajas de Ahorros a la muestra el ejercicio 2004. Así, cuatro Cajas de Ahorros pasaron a ubicarse entre las cinco primeras entidades con mayor número de mujeres en sus consejos en 2004 en el grupo de empresas no cotizadas en el mercado continuo. Si bien, esta situación privilegiada de las mujeres en los consejos de las Cajas de Ahorros respecto a otro tipo de entidades, se contrarrestó con el hecho de que también el sector financiero contó con las empresas peor situadas en cuanto a participación femenina en sus consejos.

Tabla 2. Valores extremos de la variable número de mujeres según tipo de mercado

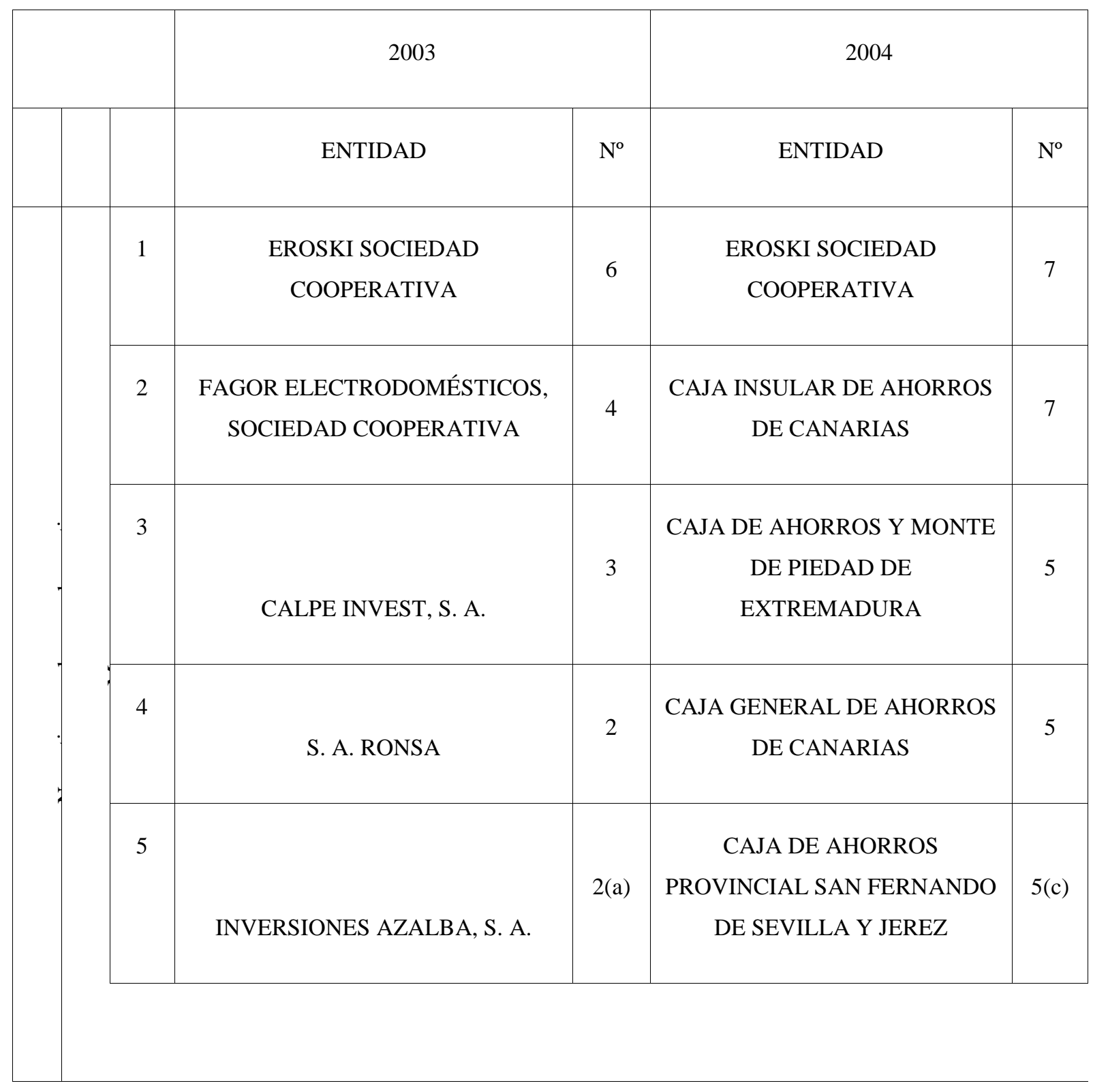

REVESCO No 110 - Primer Cuatrimestre 2013 - ISSN: 1885-8031 - www.ucm.es/info/revesco 


\begin{tabular}{|c|c|c|c|c|c|}
\hline 1 & $\begin{array}{c}\text { COMPAÑIA LOGISTICA DE } \\
\text { HIDROCARBUROS CLH, S. A. }\end{array}$ & 0 & $\begin{array}{c}\text { CAJA DE AHORROS Y MONTE } \\
\text { DE PIEDAD DE CÓRDOBA }\end{array}$ & 0 \\
\hline 2 & CARTERA HOTELERA, S. A. & 0 & $\begin{array}{c}\text { CAJA DE AHORROS Y MONTE } \\
\text { DE PIEDAD DE ÁVILA }\end{array}$ & 0 \\
\hline 3 & AGUAS DE VALENCIA S. A. & 0 & BANCO GALLEGO, S. A. & 0 \\
\hline 4 & $\begin{array}{c}\text { CAJA RURAL DE NAVARRA } \\
5\end{array}$ & $\begin{array}{c}\text { HIDROELECTRICA DEL } \\
\text { CANTABRICO, S. A. }\end{array}$ & 0 (b) & $\begin{array}{c}\text { COMPANIA LOGISTICA DE } \\
\text { HIDROCARBUROS CLH, S. A. }\end{array}$ & 0 \\
\hline
\end{tabular}

\begin{tabular}{|c|c|c|c|c|c|}
\hline 1 & TRANSPORTES AZKAR, S.A. & 4 & TRANSPORTES AZKAR, S.A. & 4 \\
\hline \multirow{2}{*}{2} & $\begin{array}{c}\text { PROSEGUR COMPANIA DE } \\
\text { SEGURIDAD, S. A. }\end{array}$ & 3 & $\begin{array}{c}\text { FOMENTO DE } \\
\text { CONSTRUCCIONES Y } \\
\text { CONTRATAS, S. A. }\end{array}$ & 4 \\
\hline 3 & BANCO DE VALENCIA & 3 & $\begin{array}{c}\text { PROSEGUR COMPANIA DE } \\
\text { SEGURIDAD, S. A. }\end{array}$ & 3 \\
\hline 4 & $\begin{array}{c}\text { FOMENTO DE CONSTRUCCIONES } \\
\text { Y CONTRATAS, S. A. }\end{array}$ & 3 & BANCO DE VALENCIA & 3 \\
\hline & $\begin{array}{c}\text { INDO INTERNACIONAL, S. A. } \\
5\end{array}$ & $2(\mathrm{a})$ & $\begin{array}{c}\text { TELEFONICA PUBLICIDAD E } \\
\text { INFORMACION, S. A. }\end{array}$ & $2(\mathrm{e})$ \\
\hline
\end{tabular}




\begin{tabular}{|c|c|c|c|c|c|}
\hline & 1 & EBRO PULEVA, S. A. & 0 & TELEFONICA, S. A. & 0 \\
\hline 2 & IBERDROLA, S. A. & 0 & UNION FENOSA, S. A. & 0 \\
\hline 3 & SOGECABLE, S. A. & 0 & METROVACESA, S. A. & 0 \\
\hline 4 & UNION FENOSA, S. A. & 0 & IBERDROLA, S. A. & 0 \\
\hline & METROVACESA, S. A. & $0(\mathrm{~b})$ & SOGECABLE, S. A. & $0(\mathrm{~d})$ \\
\hline
\end{tabular}

a En la tabla de valores extremos mayores sólo se muestra una lista parcial de los casos con el valor 2.

b En la tabla de valores extremos menores sólo se muestra una lista parcial de los casos con el valor 0 .

c En la tabla de valores extremos mayores sólo se muestra una lista parcial de los casos con el valor 5.

d En la tabla de valores extremos menores sólo se muestra una lista parcial de los casos con el valor 0.

e En la tabla de valores extremos mayores sólo se muestra una lista parcial de los casos con el valor 2.

Fuente: Elaboración propia

En la Tabla 3 figuran identificadas las entidades con el número de mujeres consejeras más alto y más bajo del total de la muestra, los dos ejercicios analizados, sin diferenciar a las empresas por grupos. Las tres entidades en los primeros niveles en el ranking respecto al número de mujeres en los consejos el año 2003 fueron las vascas: EROSKI, S. C., TRANSPORTES AZKAR, S. A., y FAGOR ELECTRODOMÉSTICOS, S. C. con seis, cuatro y cuatro mujeres en sus consejos respectivamente.

Como ya se observó previamente fue en el ejercicio 2004 en el que se alcanzaron las mayores cuotas femeninas en los consejos correspondiendo a los de las empresas EROSKI, S. C. y CAJA INSULAR DE AHORROS DE CANARIAS empatadas con siete mujeres en su consejo cada una.

Tabla 3. Valores extremos de la variable número de mujeres para toda la muestra

\begin{tabular}{|c|c|c|c|c|c|}
\hline & & 2003 & $\begin{array}{c}\text { Valor } \\
03\end{array}$ & 2004 & $\begin{array}{c}\text { Valor } \\
04\end{array}$ \\
\hline 总 & 1 & EROSKI SOCIEDAD COOPERATIVA & 6 & $\begin{array}{c}\text { EROSKI SOCIEDAD } \\
\text { COOPERATIVA }\end{array}$ & 7 \\
\hline
\end{tabular}




\begin{tabular}{|c|c|c|c|c|c|}
\hline & 2 & TRANSPORTES AZKAR, S. A. & 4 & $\begin{array}{c}\text { CAJA INSULAR DE AHORROS } \\
\text { DE CANARIAS }\end{array}$ & 7 \\
\hline & 3 & $\begin{array}{c}\text { FAGOR ELECTRODOMÉSTICOS, } \\
\text { SOCIEDAD COOPERATIVA }\end{array}$ & 4 & $\begin{array}{l}\text { CAJA DE AHORROS Y } \\
\text { MONTE DE PIEDAD DE } \\
\text { EXTREMADURA }\end{array}$ & 5 \\
\hline & 4 & CALPE INVEST, S. A. & 3 & $\begin{array}{c}\text { CAJA GENERAL DE } \\
\text { AHORROS DE CANARIAS }\end{array}$ & 5 \\
\hline & 5 & $\begin{array}{l}\text { PROSEGUR COMPANIA DE } \\
\text { SEGURIDAD, S. A. }\end{array}$ & $3(a)$ & $\begin{array}{c}\text { CAJA DE AHORROS } \\
\text { PROVINCIAL SAN } \\
\text { FERNANDO DE SEVILLA Y } \\
\text { JEREZ }\end{array}$ & $5(\mathrm{c})$ \\
\hline \multirow{5}{*}{ 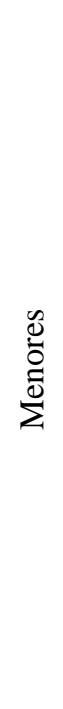 } & 1 & EBRO PULEVA, S. A. & 0 & IBERDROLA, S. A. & 0 \\
\hline & 2 & IBERDROLA, S. A. & 0 & SOGECABLE, S. A. & 0 \\
\hline & 3 & SOGECABLE, S. A. & 0 & UNION FENOSA, S. A. & 0 \\
\hline & 4 & METROVACESA, S. A. & 0 & METROVACESA, S. A. & 0 \\
\hline & 5 & $\begin{array}{l}\text { COMPAÑIA LOGISTICA } \\
\text { HIDROCARBUROS CLH S. A. }\end{array}$ & $0(b)$ & $\begin{array}{l}\text { COMPAÑIA LOGISTICA DE } \\
\text { HIDROCARBUROS CLH, S. A. }\end{array}$ & $0(\mathrm{~d})$ \\
\hline
\end{tabular}

a En la tabla de valores extremos mayores sólo se muestra una lista parcial de los casos con el valor 3.

b En la tabla de valores extremos menores sólo se muestra una lista parcial de los casos con el valor 0 .

c En la tabla de valores extremos mayores sólo se muestra una lista parcial de los casos con el valor 5.

d En la tabla de valores extremos menores sólo se muestra una lista parcial de los casos con el valor 0.

Fuente: Elaboración propia

\subsubsection{Valores extremos: porcentaje de mujeres}

Al tomar los datos correspondientes al porcentaje de mujeres en cada consejo, EROSKI, que el año 2003 en cuanto a número de mujeres en los análisis anteriores ocupaba el primer lugar, el año 2004 y aunque no aparece en la lista de las cinco primeras, empataría 
con S. A. RONSA, INVERSIONES AZALBA, S. A. e INDUSTRIAS DEL CURTIDO, S. A. con una participación femenina paritaria a la de sus homólogos masculinos (Tabla 4). Ambos ejercicios la entidad INMUEBLES DE CAJA HIPOTECARIA CATALANA MUTUAL, S.A. ocupó el primer puesto, si bien, por su carácter unipersonal habría que valorar la conveniencia de eliminarla dadas sus características en determinados análisis por ser un valor extremo. Una vez eliminada, sería la inmobiliaria castellonense CALPE INVEST, S. A. la que se posicionaría en primer lugar. Además, tanto CALPE INVEST, S. A. como EROSKI evolucionaron de manera más beneficiosa para las mujeres al incrementar su participación en 2004 respecto al año anterior. Esta tendencia positiva en las empresas más propicias a las mujeres podría estar en consonancia con las posibles razones planteadas por Farrell y Hersch (2005) por las cuales la participación femenina en los consejos podría tener un efecto positivo sobre la incorporación de otras mujeres. Los autores sugieren que la existencia de miembros femeninos en los consejos podría tener influencia a la hora de atraer a mujeres adicionales para servir en los consejos, de identificar homólogas para nominaciones o de dar respuesta a la presión externa. También señalan los autores que la nominación de mujeres puede ser más atractiva en aquellas firmas que han logrado algún grado de diversidad en sus consejos.

Tabla 4. Valores extremos de la variable porcentaje de mujeres por tipo de mercado

\begin{tabular}{|c|c|c|c|c|c|}
\hline & & \multicolumn{2}{|l|}{2003} & \multicolumn{2}{|l|}{2004} \\
\hline & & ENTIDAD & $\%$ & ENTIDAD & $\%$ \\
\hline \multirow{4}{*}{ 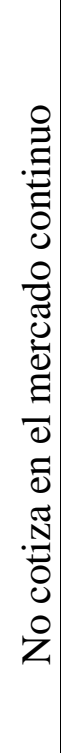 } & 1 & $\begin{array}{c}\text { INMUEBLES DE CAJA } \\
\text { HIPOTECARIA CATALANA } \\
\text { MUTUAL, S. A. (PLARREGA } \\
\text { INVEST 2000, S. A.) }\end{array}$ & 100 & $\begin{array}{c}\text { INMUEBLES DE CAJA } \\
\text { HIPOTECARIA CATALANA } \\
\text { MUTUAL, S. A. (PLARREGA } \\
\text { INVEST 2000, S. A.) }\end{array}$ & 100 \\
\hline & 2 & CALPE INVEST, S. A. & 60 & CALPE INVEST, S. A. & 80 \\
\hline & 3 & $\begin{array}{l}\text { EROSKI SOCIEDAD } \\
\text { COOPERATIVA }\end{array}$ & 50 & $\begin{array}{c}\text { EROSKI SOCIEDAD } \\
\text { COOPERATIVA }\end{array}$ & 58 \\
\hline & 4 & S. A. RONSA & 50 & S. A. RONSA & 50 \\
\hline
\end{tabular}

REVESCO No 110 - Primer Cuatrimestre 2013 - ISSN: 1885-8031 - www.ucm.es/info/revesco 


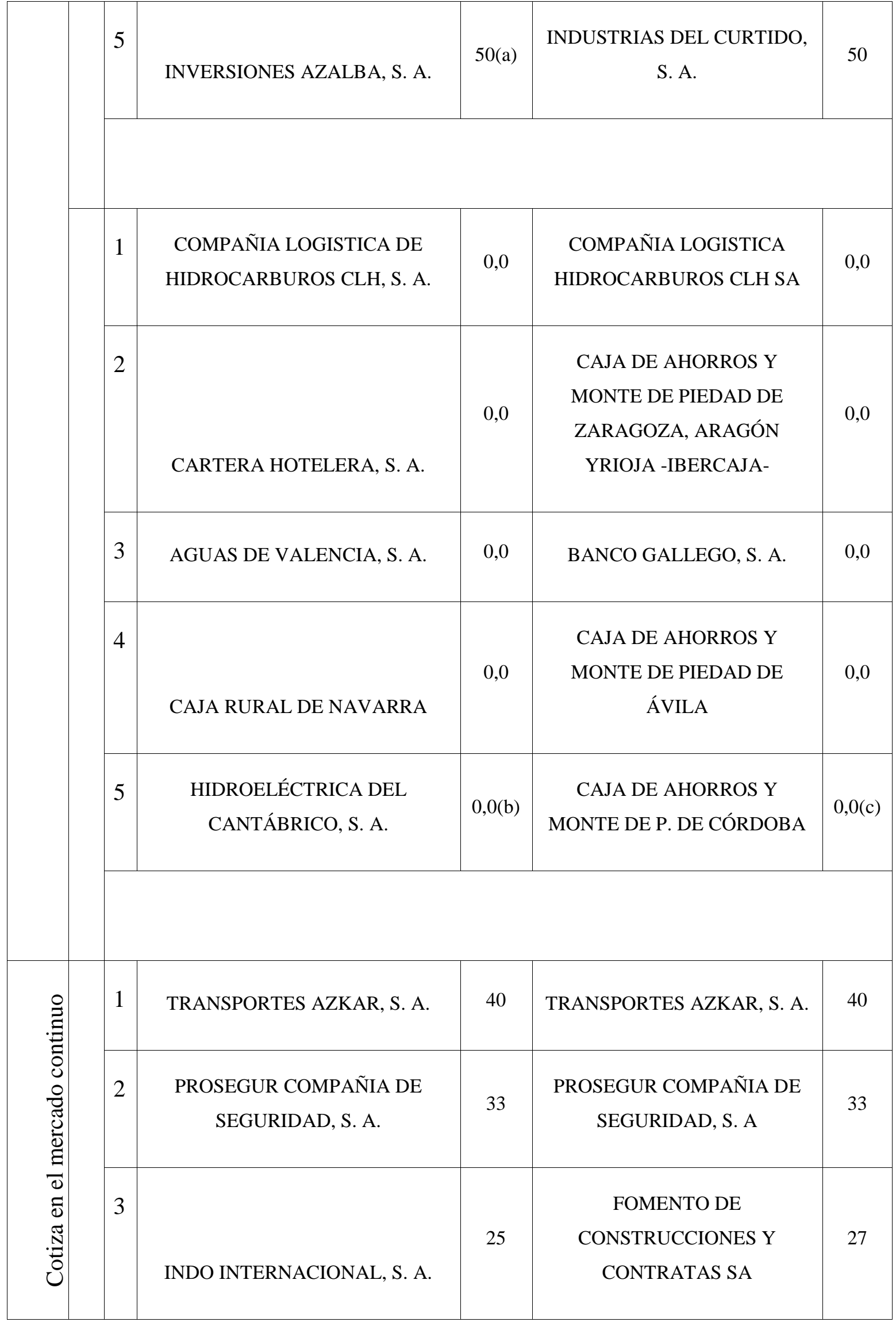




\begin{tabular}{|c|c|c|c|c|}
\hline 4 & $\begin{array}{l}\text { TELEFÓNICA PUBLICIDAD E } \\
\text { INFORMACIÓN, S. A. }\end{array}$ & 25 & $\begin{array}{l}\text { TELEFÓNICA PUBLICIDAD E } \\
\text { INFORMACIÓN, S. A. }\end{array}$ & 25 \\
\hline 5 & NATRACEUTICAL, S. A. & 25 & $\begin{array}{c}\text { SOCIEDAD ESP. DEL } \\
\text { ACUMULADOR TUDOR SA }\end{array}$ & $25(\mathrm{~d})$ \\
\hline 1 & EBRO PULEVA, S. A. & 0,0 & IBERDROLA, S. A. & 0,0 \\
\hline 2 & IBERDROLA, S. A. & 0,0 & SOGECABLE, S. A. & 0,0 \\
\hline 3 & SOGECABLE, S. A. & 0,0 & UNIÓN FENOSA, S. A. & 0,0 \\
\hline 4 & UNIÓN FENOSA, S. A. & 0,0 & METROVACESA, S. A. & 0,0 \\
\hline 5 & METROVACESA, S. A. & $0,0(\mathrm{~b})$ & TELEFÓNICA, S. A.. & $0,0(\mathrm{c})$ \\
\hline
\end{tabular}

a En la tabla de valores extremos mayores sólo se muestra una lista parcial de los casos con el valor 50. b En la tabla de valores extremos menores sólo se muestra una lista parcial de los casos con el valor 0,0 .

c En la tabla de valores extremos menores sólo se muestra una lista parcial de los casos con el valor 0,0 . d En la tabla de valores extremos mayores sólo se muestra una lista parcial de los casos con el valor 25.

\section{Fuente: Elaboración propia}

Al tomar los valores extremos diferenciados por mercado de la muestra completa para los dos ejercicios se puede comprobar de nuevo que fueron las entidades no cotizadas en el mercado continuo las que ocuparon los primeros puestos en participación femenina tanto en el ejercicio 2003 como en el 2004 (Tabla 5).

Tabla 5. Valores extremos de la variable porcentaje de mujeres para toda la muestra

\begin{tabular}{|l|l|l|l|l|l|}
\hline & 2003 & $\%$ & 2004 & $\%$ \\
\hline
\end{tabular}




\begin{tabular}{|c|c|c|c|c|}
\hline 1 & $\begin{array}{c}\text { INMUEBLES DE CAJA HIPOTECARIA } \\
\text { CATALANA MUTUAL, S. A. } \\
\text { (PLARREGA INVEST, 2000, S.A.) }\end{array}$ & 100 & $\begin{array}{c}\text { INMUEBLES DE CAJA } \\
\text { HIPOTECARIA CATALANA } \\
\text { MUTUAL, S. A. (PLARREGA INVEST, } \\
2000, \text { S. A.) }\end{array}$ & 100 \\
\hline 2 & CALPE INVEST, S. A. & 60 & CALPE INVEST, S. A. & 80 \\
\hline 3 & EROSKI SOCIEDAD COOPERATIVA & 50 & EROSKI SOCIEDAD COOPERATIVA & 58 \\
\hline 4 & S. A. RONSA & 50 & S. A. RONSA & 50 \\
\hline 5 & INVERSIONES AZALBA, S. A. & $50(a)$ & INDUSTRIAS DEL CURTIDO, S. A. & 50 \\
\hline 1 & EBRO PULEVA, S. A. & 0,0 & IBERDROLA, S. A. & 0,0 \\
\hline 2 & IBERDROLA, S. A. & 0,0 & SOGECABLE, S. A. & 0,0 \\
\hline 3 & SOGECABLE, S. A. & 0,0 & UNIÓN FENOSA, S. A. & 0,0 \\
\hline 4 & METROVACESA, S. A. & 0,0 & METROVACESA, S. A. & 0,0 \\
\hline 5 & $\begin{array}{l}\text { COMPAÑIA LOGISTICA } \\
\text { HIDROCARBUROS CLH SA }\end{array}$ & $0,0(\mathrm{~b})$ & $\begin{array}{l}\text { COMPAÑIA LOGISTICA } \\
\text { HIDROCARBUROS CLH S A }\end{array}$ & $0,0(\mathrm{c})$ \\
\hline
\end{tabular}

a En la tabla de valores extremos mayores sólo se muestra una lista parcial de los casos con el valor 50.

b En la tabla de valores extremos menores sólo se muestra una lista parcial de los casos con el valor 0,0 .

c En la tabla de valores extremos menores sólo se muestra una lista parcial de los casos con el valor 0,0 .

Fuente: Elaboración propia

\subsection{Relaciones entre rentabilidad y género en los consejos}

\subsubsection{Mercado continuo y no continuo}

En concordancia con los objetivos del trabajo se muestran los resultados de poner en correspondencia la medida contable rentabilidad económica con cada uno de los dos indicadores que reflejan la participación femenina en los consejos mediante el análisis de la 
varianza. Primero se observan las relaciones entre la media de la rentabilidad económica en los consejos y el número de mujeres y después se comparan con el porcentaje de mujeres.

\section{Relación entre la rentabilidad económica y el número de mujeres}

Como se puede apreciar en la Tabla 6, la media de la rentabilidad económica de entidades tanto cotizadas como no cotizadas en el mercado continuo es del 5,97\%. El valor mínimo se corresponde con una entidad del sector inmobiliario con una mujer en un consejo de nueve miembros, y el máximo en la única entidad con siete mujeres en su consejo perteneciente al sector de bienes de consumo, y forma jurídica de sociedad cooperativa.

Tabla 6. Estadísticos descriptivos. número de mujeres. RE: Mercado continuo y no continuo

\begin{tabular}{|c|c|c|c|c|c|c|c|c|c|c|}
\hline & & $\mathrm{N}$ & Media & $\begin{array}{l}\text { Desviación } \\
\text { típica }\end{array}$ & $\begin{array}{l}\text { Error } \\
\text { típico }\end{array}$ & \multicolumn{2}{|c|}{$\begin{array}{l}\text { Intervalo de } \\
\text { confianza para la } \\
\text { media al } 95 \%\end{array}$} & \multirow[t]{2}{*}{ Mínimo } & \multirow[t]{2}{*}{ Máximo } & \multirow[t]{2}{*}{$\begin{array}{l}\text { Varianza } \\
\text { entre } \\
\text { comp. }\end{array}$} \\
\hline & & & & & & $\begin{array}{l}\text { Lím. } \\
\text { Inf. }\end{array}$ & $\begin{array}{l}\text { Lím. } \\
\text { sup. }\end{array}$ & & & \\
\hline $\mathrm{RE}$ & 0 & 92 & 4,83 & 14,08 & 1,46 & 1,92 & 7,75 & $-91,93$ & 40,42 & \\
\hline & 1 & 42 & 5,92 & 5,98 & 0,92 & 4,06 & 7,78 & $-7,92$ & 18,12 & \\
\hline & 2 & 14 & 10,27 & 8,52 & 2,28 & 5,35 & 15,19 & 1,44 & 28,85 & \\
\hline & 3 & 1 & 9,09 & & & & & 9,09 & 9,09 & \\
\hline & 4 & 4 & 5,50 & 3,00 & 1,50 & 0,72 & 10,28 & 2,52 & 8,51 & \\
\hline & 7 & 1 & 50,79 & & & & & 50,79 & 50,79 & \\
\hline & Total & 154 & 5,97 & 12,23 & 0,98 & 4,02 & 7,92 & $-91,9$ & 50,79 & \\
\hline
\end{tabular}




\begin{tabular}{|c|c|c|c|c|c|c|}
\hline Mod & $\begin{array}{l}\text { Ef. } \\
\text { fijos }\end{array}$ & 11,76 & 0,95 & 4,09 & 7,84 & \\
\hline & Ef. & & 3,09 & $-1,99$ & 13,94 & 19,77 \\
\hline & al. & & & & & \\
\hline
\end{tabular}

a Advertencia: La varianza entre componentes es negativa. Ha sido reemplazada por 0 al calcular esta medida para los efectos Aleatorios

Fuente: Elaboración propia

La media de la rentabilidad económica más baja, del 4,84\%, se obtiene en las empresas sin presencia femenina, mientras que la única empresa con siete mujeres alcanza la más alta, del 50,79\%. Los consejos con una media de la rentabilidad económica inferior a la del total de la muestra, además de los 92 que prescinden de mujeres, son los que cuentan con cuatro consejeras con una media de la rentabilidad del 5,50\% y los de una única mujer en sus consejos con un 5,92\% de rentabilidad. Todos los demás están por encima de la media, con rentabilidad media por orden decreciente del 10,26\% en los consejos con dos mujeres, y del 9,09\% en el único con tres. Las cifras más bajas para los consejos con presencia femenina se dan en los de cuatro mujeres donde la rentabilidad está por debajo de la media siendo del $5,5 \%$, y en los de una única mujer consejera que es del 5,92\%.

El estadístico de Levene sobre igualdad de varianzas aporta un valor de 0,46 y un pvalor de 0,71 , lo que lleva a aceptar la hipótesis nula de igualdad de varianzas y a concluir que en las poblaciones definidas por los siete tipos de participación femenina las varianzas de la variable rentabilidad económica son iguales (Tabla 7). 
Tabla 7. Prueba de homogeneidad de varianzas. $n^{\circ}$ mujeres. re: Mercado continuo y no continuo

\begin{tabular}{|l|c|c|c|c|}
\hline & Estadístico de Levene & gl1 & g12 & Sig. \\
\hline RE & $0,46(\mathrm{a})$ & 3 & 148 & 0.71 \\
\hline
\end{tabular}

a Groups with only one case are ignored in computing the test of homogeneity of variance for Rentabilidad Económica

\section{Fuente: Elaboración propia}

Con la finalidad de analizar la potencia en las relaciones entre la media de la rentabilidad económica de las empresas y la participación femenina medida por el número de mujeres en los consejos se realiza un análisis de la varianza de un factor (Tabla 8). El nivel crítico asociado al estadístico $\mathrm{F}=3,46$ es igual a 0,005 por lo que se rechaza la hipótesis de igualdad de medias, lo que significa que las medias poblacionales comparadas no son iguales, o lo que es lo mismo, se observan diferencias estadísticamente significativas en la media de la rentabilidad económica en función del número de mujeres en los consejos.

Tabla 8. Anova de un factor de la RE y el $n^{o}$. de mujeres: Mercado continuo y no continuo

\begin{tabular}{|c|c|c|c|c|c|c|}
\hline & & $\begin{array}{c}\text { Suma de } \\
\text { cuadrados }\end{array}$ & Gl & Media cuadrática & F & Sig. \\
\hline \multirow{2}{*}{ RE } & Inter-grupos & 2396,60 & 5 & 479,32 & 3,46 & 0,005 \\
\cline { 2 - 7 } & Intra-grupos & 20482,14 & 148 & 138,39 & & \\
\cline { 2 - 7 } & Total & 22878,74 & 153 & & & \\
\hline
\end{tabular}

Fuente: Elaboración propia

Para comprobar si estos resultados se deben al valor representado por la única empresa con siete mujeres consejeras con la mayor rentabilidad económica de la muestra se repiten los 
análisis eliminando esta entidad (Tabla 9) y efectivamente se obtiene que la media de la rentabilidad económica disminuye pasando a ser del 5,68\%, mas baja que la conseguida cuando se incluyen a todas las entidades que asciende al 5,97\%.

Tabla 9. Estadísticos descriptivos re una vez eliminada a la única entidad con 7 mujeres en sus consejos: Mercado continuo y no continuo

\begin{tabular}{|c|c|c|c|c|c|c|c|c|c|c|}
\hline & & $\mathrm{N}$ & Media & $\begin{array}{c}\text { Desviación } \\
\text { típica }\end{array}$ & $\begin{array}{l}\text { Error } \\
\text { típico }\end{array}$ & \multicolumn{2}{|c|}{$\begin{array}{c}\text { I. C. para la media } \\
\text { al } 95 \%\end{array}$} & \multirow[t]{2}{*}{ Mínimo } & \multirow[t]{2}{*}{ Máximo } & \multirow[t]{2}{*}{$\begin{array}{l}\text { Varianza } \\
\text { entre comp. }\end{array}$} \\
\hline & & & & & & Lím. Inf. & Lím. Sup & & & \\
\hline 0 & & 92 & 4,84 & 14,08 & 1,47 & 1,92 & 7,75 & $-91,93$ & 40,42 & \\
\hline 1 & & 42 & 5,92 & 5,98 & 0,92 & 4,06 & 7,79 & $-7,92$ & 18,12 & \\
\hline 2 & & 14 & 10,27 & 8,52 & 2,28 & 5,35 & 15,19 & 1,44 & 28,85 & \\
\hline 3 & & 1 & 9,09 & & & & & 9,09 & 9.09 & \\
\hline 4 & & 4 & 5,50 & 3,00 & 1,50 & 0,72 & 10,28 & 2,52 & 8,51 & \\
\hline Total & & 153 & 5,68 & 11,71 & 0,95 & 3,81 & 7,55 & $-91,93$ & 40,42 & \\
\hline Mod & Ef.fijos & & & 11,76 & 0,95 & 3,80 & 7,56 & & & \\
\hline & Ef.aleat. & & & & $0,95(a)$ & $3,03(\mathrm{a})$ & $8,32(a)$ & & & $-2,11$ \\
\hline
\end{tabular}

a Advertencia: La varianza entre componentes es negativa. Ha sido reemplazada por 0.0 al calcular esta medida para los efectos aleatorios.

Fuente: Elaboración propia

En lo que respecta al análisis de la varianza, el nivel crítico asociado al estadístico $\mathrm{F}=0,68$ es igual a 0,61 por lo que al no haber diferencias estadísticamente significativas en los 
diferentes consejos clasificados según el número de mujeres se acepta la hipótesis nula, en consecuencia se puede afirmar que una única entidad, la sociedad cooperativa EROSKI, contribuye a cambiar los resultados relativos a la igualdad de medias (Tabla 10).

Tabla 10. Anova de la RE una vez eliminada a la única entidad con 7 mujeres en sus consejos: Mercado continuo y no continuo

\begin{tabular}{|l|c|c|c|c|c|}
\hline & $\begin{array}{c}\text { Suma de } \\
\text { cuadrados }\end{array}$ & Gl & $\begin{array}{c}\text { Media } \\
\text { cuadrática }\end{array}$ & F & Sig. \\
\hline Inter-grupos & 374.64 & 4,00 & 93,66 & 0,68 & 0,61 \\
\hline Intra-grupos & 20482.14 & 148,00 & 138,39 & & \\
\hline Total & 20856.78 & 152,00 & & & \\
\hline
\end{tabular}

Fuente: Elaboración propia

Esta situación podría colocar a esta empresa como modelo de estudio de cómo una entidad con una presencia femenina elevada en su consejo obtiene una rentabilidad económica muy superior a la del resto de entidades. No es posible realizar las pruebas robustas de la igualdad de medias porque al menos un grupo tiene una suma de ponderaciones de los casos menor o igual que 1 . Tampoco se pueden realizar pruebas post hoc debido a la existencia de grupos con menos de dos casos.

\section{Relación entre la rentabilidad económica y el porcentaje de mujeres}

En cuanto a los resultados de los estadísticos descriptivos de la media de la rentabilidad económica en relación con el porcentaje de mujeres de los consejos después de haber sido codificado para poder aplicar los estadísticos, se observa que en los 92 consejos sin presencia femenina la media de la rentabilidad económica es del 4,84\%, cifra inferior a la de la media para toda la muestra que es del 5,97\% (Tabla 11). 
Tabla 11. Estadísticos descriptivos. porcentaje de mujeres. RE: Mercado continuo y no continuo

\begin{tabular}{|c|c|c|c|c|c|c|c|c|c|}
\hline & $\mathrm{N}$ & Media & Desv. & $\begin{array}{l}\text { Error } \\
\text { típico }\end{array}$ & \multicolumn{2}{|c|}{$\begin{array}{c}\text { I. C. para la media } \\
\text { al } 95 \%\end{array}$} & Mín & Máx & $\begin{array}{l}\text { Var. } \\
\text { comp. }\end{array}$ \\
\hline & & & & & Lím. Inf. & $\begin{array}{l}\text { Lím. } \\
\text { Sup }\end{array}$ & & & \\
\hline 0 (sin mujeres) & 92 & 4,84 & 14,08 & 1,47 & 1,92 & 7,75 & $-91,90$ & 40,42 & \\
\hline $1(0 \%<\%$ mujeres $<10 \%)$ & 19 & 8,11 & 5,68 & 1,30 & 5,37 & 10,85 & $-1,29$ & 18,12 & \\
\hline $2(10 \% \leq \%$ mujeres $<20 \%)$ & 22 & 6,28 & 6,98 & 1,49 & 3,19 & 9,38 & $-7,92$ & 21,10 & \\
\hline $3(20 \% \leq \%$ mujeres $<50 \%)$ & 16 & 7,25 & 8,03 & 2,01 & 2,97 & 11,53 & $-4,90$ & 28,85 & \\
\hline 4 (\%mujeres $\geq 50 \%$ & 5 & 13,23 & 21,02 & 9,40 & $-12,88$ & 39,33 & 2,55 & 50,79 & \\
\hline Total & 154 & 5,97 & 12,23 & 0,99 & 4,02 & 7,92 & $-91,90$ & 50,79 & \\
\hline Mod. Ef.. fijos & & & 12,26 & 0,99 & 4,02 & 7,92 & & & \\
\hline
\end{tabular}

a Advertencia: La varianza entre componentes es negativa. Ha sido reemplazada por 0,0 al calcular esta medida para los efectos aleatorios.

Fuente: Elaboración propia

La media de la rentabilidad económica máxima se alcanza en los consejos con más del $50 \%$ de mujeres, mientras que la mínima se obtiene en los consejos sin presencia femenina. Excepto en los consejos sin presencia femenina, en todos los demás la media de la rentabilidad económica en cada uno de ellos es superior a la media para toda la muestra.

Con el contraste de Levene de homogeneidad de varianzas de la media de la rentabilidad económica no se obtienen diferencias estadísticamente significativas entre los 
distintos tipos de consejos codificados por lo que se acepta la hipótesis nula de igualdad de varianzas entre los distintos intervalos definidos de porcentaje de mujeres (Tabla 12).

Tabla 12. Prueba de homogeneidad de varianzas. porcentaje de mujeres. RE: Mercado continuo y no continuo

\begin{tabular}{|l|c|c|c|c|}
\hline & Estadístico de Levene & gl1 & g12 & Sig. \\
\hline $\mathrm{RE}$ & 1,06 & 4 & 149 & 0,39 \\
\hline
\end{tabular}

Fuente: Elaboración propia

La prueba Anova de un factor aporta un estadístico de $\mathrm{F}=0,83$ con un p-valor de 0,51 (Tabla 13) aceptándose por tanto la hipótesis nula de igualdad de medias.

Tabla 13. Anova de un factor de la RE y el porcentaje de mujeres: Mercado continuo y no continuo

\begin{tabular}{|l|c|c|c|c|c|c|}
\hline & & $\begin{array}{c}\text { Suma de } \\
\text { cuadrados }\end{array}$ & Gl & $\begin{array}{c}\text { Media } \\
\text { cuadrática }\end{array}$ & F & Sig. \\
\hline RE & Inter-grupos & 496,91 & 4,00 & 124,23 & 0,83 & 0,51 \\
\cline { 2 - 7 } & Intra-grupos & 22381,83 & 149,00 & 150,21 & & \\
\hline
\end{tabular}

Fuente: Elaboración propia

\subsubsection{Mercado continuo}

En este apartado se sigue la misma metodología que en el anterior en el que se ha analizado la rentabilidad económica para entidades cotizadas y no cotizadas en el mercado continuo pero únicamente para la muestra de sociedades cotizadas en el mercado continuo. En este caso, al eliminar de la muestra, entre otras, a la entidad cooperativa EROSKI por no cotizar en el mencionado mercado, y ser la única entidad con siete mujeres, el máximo número de mujeres en un consejo en la nueva muestra es de cuatro, aspecto que es preciso tener en cuenta a la hora de interpretar los resultados. 


\section{Relación entre la rentabilidad económica y el número de mujeres}

La media de la rentabilidad económica de las entidades del mercado continuo es del $6,20 \%$, siendo la de los consejos sin presencia femenina la menor, alcanzando un valor de $5,31 \%$, seguida de la de consejos con una única mujer entre sus filas con un valor ligeramente superior a la media, alcanzando una cifra de 6,34\% (Tabla 14). Son los consejos con dos mujeres los de mayor rentabilidad económica con una media del 18,44\%, seguidos de los de tres mujeres con un valor de $9,09 \%$, y de los de cuatro con una media del $8,06 \%$.

Tabla 14. Estadísticos descriptivos. número de mujeres. RE: Mercado continuo

\begin{tabular}{|c|c|c|c|c|c|c|c|c|c|c|}
\hline & & $\mathrm{N}$ & Media & Desv. & $\begin{array}{l}\text { Error } \\
\text { típico }\end{array}$ & \multicolumn{2}{|c|}{$\begin{array}{l}\text { I.C. para la media al } \\
\qquad 95 \%\end{array}$} & Mín. & Máx. & $\begin{array}{l}\text { Var. } \\
\text { comp }\end{array}$ \\
\hline & & & & & & \multicolumn{2}{|c|}{ Límite } & & & \\
\hline & & & & & & inferior & superior & & & \\
\hline 0 & & 67 & 5,31 & 16,24 & 1,98 & 1,35 & 9,27 & $-91,9$ & 40,42 & \\
\hline 1 & & 30 & 6,34 & 6,57 & 1,20 & 3,88 & 8,79 & $-7,92$ & 18,12 & \\
\hline 2 & & 4 & 18,44 & 8,75 & 4,37 & 4,53 & 32,36 & 9,08 & 28,85 & \\
\hline 3 & & 1 & 9,09 & , & , & , & , & 9,09 & 9,09 & \\
\hline 4 & & 2 & 8,07 & 0,63 & 0,45 & 2,41 & 13,72 & 7,62 & 8,51 & \\
\hline Total & & 104 & 6,20 & 13,78 & 1,35 & 3,52 & 8,88 & $-91,9$ & 40,42 & \\
\hline Mod & Ef fijos & & & 13,81 & 1,35 & 3,51 & 8,89 & & & \\
\hline
\end{tabular}




\begin{tabular}{l|l|l|l|l|l|l|l|l|} 
Ef. aleat. & & & & & & & & \\
\end{tabular}

a Advertencia: La varianza entre componentes es negativa. Ha sido reemplazada por 0,0 al calcular esta medida para los efectos aleatorios.

Fuente: Elaboración propia

La prueba de homogeneidad de varianzas realizada utilizando el Estadístico de Levene no muestra diferencias estadísticamente significativas en la rentabilidad económica media (Tabla 15), del mismo modo que la prueba Anova de un factor manifiesta que no existen diferencias estadísticamente significativas entre las medias de la rentabilidad económica y del número de mujeres en los consejos (Tabla 16).

Tabla 15. Prueba de homogeneidad de varianzas. $n^{\circ}$ mujeres. RE: Mercado continuo

\begin{tabular}{|l|l|l|l|l|}
\hline & Estadístico de Levene & gl1 & g12 & Sig. \\
\hline Rentabilidad Económica & $0,32(\mathrm{a})$ & 3 & 99 & 0,81 \\
\hline
\end{tabular}

a Groups with only one case are ignored in computing the test of homogeneity of variance for RE

\section{Fuente: Elaboración propia}

Tabla 16. Anova de un factor de la RE y el $n^{\circ}$. de mujeres: Mercado continuo

\begin{tabular}{|l|l|c|c|c|c|c|}
\hline \multicolumn{2}{|l|}{} & $\begin{array}{c}\text { Suma de } \\
\text { cuad. }\end{array}$ & gl & $\begin{array}{c}\text { Media } \\
\text { cuadrát }\end{array}$ & F & Sig. \\
\hline \multirow{2}{*}{$\begin{array}{l}\text { Rentabilidad } \\
\text { económica }\end{array}$} & Inter-grupos & 668,81 & 4,00 & 167,20 & 0,88 & 0,48 \\
\cline { 2 - 8 } & Intra-grupos & 18884,04 & 99,00 & 190,75 & & \\
\cline { 2 - 8 } & Total & 19552,86 & 103,00 & & & \\
\hline
\end{tabular}

Fuente: Elaboración propia 


\section{Relación entre la rentabilidad económica y el porcentaje de mujeres}

La media de la rentabilidad económica para el conjunto de empresas cotizadas en el mercado continuo que forman parte de la muestra es del 6,20\% (Tabla 17). Cabe señalar que tras la eliminación de las entidades no cotizadas en el mercado continuo, en ningún consejo se alcanza paridad con respecto al género ni mayor porcentaje de mujeres que de hombres. La media más alta se da en aquellos consejos con un porcentaje de mujeres mayor o igual que el $20 \%$ y menor del $50 \%$ con una media de la rentabilidad económica del $12,05 \%$, casi el doble que la de las empresas sin presencia femenina. La siguiente media del 8,20\% se observó en los consejos con presencia femenina inferior al $10 \%$ pero al menos una mujer. Los consejos con un porcentaje de mujeres inferior al $20 \%$ pero igual o superior al $10 \%$ alcanzan una media de la rentabilidad económica del 5,25\%, cifra por debajo de la media.

Tabla 17. Estadísticos descriptivos. porcentaje de mujeres. RE: Mercado continuo

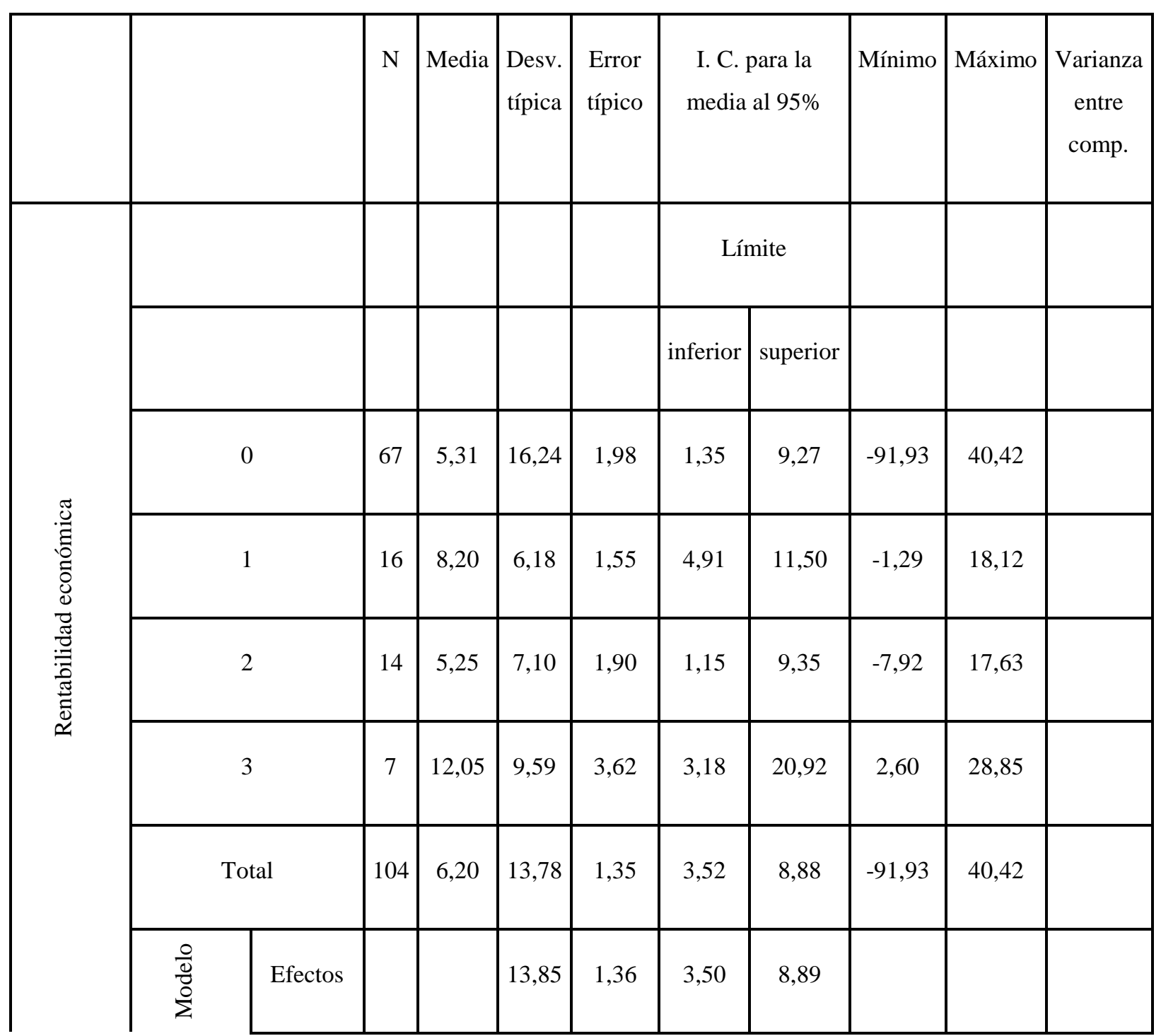

REVESCO No 110 - Primer Cuatrimestre 2013 - ISSN: 1885-8031 - www.ucm.es/info/revesco 


\begin{tabular}{|c|c|c|c|c|c|c|c|c|c|c|}
\hline fijos & & & & & & & & & \\
\hline & $\begin{array}{c}\text { Ef } \\
\text { aleatorios }\end{array}$ & & & & $1,35(\mathrm{a})$ & $1,87(\mathrm{a})$ & $10,52(\mathrm{a})$ & & & $-3,67$ \\
\hline
\end{tabular}

a Advertencia: La varianza entre componentes es negativa. Ha sido reemplazada por 0,0 al calcular esta medida para los efectos aleatorios.

Fuente: Elaboración propia

La prueba de homogeneidad de varianzas no muestra diferencias estadísticamente significativas en la rentabilidad económica media (Tabla 18), del mismo modo que la prueba Anova de un factor no revela diferencias entre las medias de la rentabilidad económica y del porcentaje de mujeres en los consejos (Tabla 19).

Tabla 18. Prueba de homogeneidad de varianzas. porcentaje mujeres. RE: Mercado continuo

\begin{tabular}{|l|l|l|l|l|}
\hline & Estadístico de Levene & gl1 & gl2 & Sig. \\
\hline Rentabilidad Económica & 0.160 & 3 & 100 & 0,923 \\
\hline
\end{tabular}

Fuente: Elaboración propia

Tabla 19 Anova de un factor de la RE y el porcentaje de mujeres: Mercado continuo

\begin{tabular}{|l|c|c|c|c|c|c|}
\hline \multicolumn{2}{|l|}{} & $\begin{array}{c}\text { Suma de } \\
\text { cuad. }\end{array}$ & $\mathrm{gl}$ & $\begin{array}{c}\text { Media } \\
\text { cuadrát }\end{array}$ & $\mathrm{F}$ & Sig. \\
\hline $\begin{array}{l}\text { Rentabilidad } \\
\text { económica }\end{array}$ & Inter-grupos & 369,74 & 3 & 123,25 & 0,642 & 0,58 \\
\cline { 2 - 7 } & Intra-grupos & 19183,11 & 100 & 191,83 & & \\
\cline { 2 - 7 } & Total & 19552,85 & 103 & & & \\
\hline
\end{tabular}

Fuente: Elaboración propia 


\section{CONCLUSIONES}

En el presente trabajo se ha puesto de manifiesto el posicionamiento de las empresas españolas obligadas a presentar un Informe Anual sobre Gobierno Corporativo a la Comisión Nacional del Mercado de Valores, en un periodo previo a la publicación del Código Unificado de Buen Gobierno y a la Ley de Igualdad, con en fin de analizar la situación de las mujeres en momento en el que no existían recomendaciones sobre paridad y por lo tanto las empresas no estaban condicionadas a seguir estas medidas. Posteriormente se ha analizado la relación existente entre la presencia femenina en los consejos de administración, y/o rectores en el caso de las cooperativas, y la rentabilidad de las entidades españolas más influyentes.

Los resultados muestran que los dos ejercicios analizados las empresas con consejos más favorables a las mujeres radicaron en el País Vasco. El año 2003, las dos entidades en las primeras posiciones en el ranking respecto al número de mujeres fueron EROSKI SOCIEDAD COOPERATIVA y FAGOR ELECTRODOMÉSTICOS, SOCIEDAD COOPERATIVA con seis y cuatro mujeres en sus consejos respectivamente. Es importante señalar que la forma jurídica de ambas entidades se diferencia de la mayoría de las empresas de la muestra por tratarse de sociedades cooperativas. El ejercicio 2004, EROSKI SOCIEDAD COOPERATIVA y CAJA INSULAR DE AHORROS DE CANARIAS fueron las entidades mejor posicionadas en cuanto a número de mujeres, empatadas, con siete mujeres en su consejo cada una. Se aprecian diferencias entre los ejercicios 2003 y 2004 debidas a la incorporación de las Cajas de Ahorros a la muestra el ejercicio 2004. Así, cuatro Cajas de Ahorros pasaron a ubicarse entre las cinco primeras entidades con mayor número de mujeres en sus consejos en 2004 en el grupo de empresas no cotizadas en el mercado continuo. Si bien, esta situación privilegiada de las mujeres en los consejos de las Cajas de Ahorros respecto a otro tipo de entidades, se contrarrestó con el hecho de que también el sector financiero contó con las empresas peor situadas en cuanto a participación femenina en sus consejos. Si en lugar de tomar los datos correspondientes al número se utilizan los relativos al porcentaje de mujeres se aprecia que la entidad cooperativa EROSKI evolucionó de manera más beneficiosa para las mujeres al incrementar su participación en 2004 respecto al año anterior.

En cuanto a las relaciones del género en los consejos con la rentabilidad económica, en un primer análisis en el que en la muestra se incluían a entidades cotizadas y no cotizadas en el mercado continuo, en el caso de éstas últimas obligadas a presentar por primera vez un 
IAGC a la CNMV, una vez eliminadas a las entidades financieras y de seguros, los resultados sugieren que, en general, la presencia femenina es mayor en el caso de las empresas más rentables. Para comprobar si estos resultados venían determinados por la presencia en la muestra de una empresa con siete mujeres en su consejo y con la mayor rentabilidad económica se repiten los análisis excluyendo a la mencionada compañía. Los resultados indican que tras la eliminación de esta empresa, las diferencias dejan de ser significativas, lo que evidencia que una única entidad contribuyó a cambiar los resultados y por lo tanto podría constituir un modelo de estudio de cómo una empresa con una elevada presencia femenina en su consejo destaca sobre todas las demás por alcanzar la mayor rentabilidad económica posicionándose en primer lugar. Una característica diferenciadora de esta empresa es su forma jurídica, al tratarse de una empresa cooperativa. En una segunda exploración se excluyen de la muestra a las sociedades no cotizadas en el mercado continuo con el fin de poder repetir los análisis anteriores. Aunque por lo general la rentabilidad fue mayor en las empresas con mayor presencia femenina no se observan diferencias estadísticamente significativas para este grupo de empresas. Los resultados anteriores revelan que la presencia femenina es mayor cuando se incluyen en la muestra a las entidades no cotizadas en el mercado continuo obligadas a presentar un IAGC, lo que podría tener relación con la estructura de capital de estas empresas.

\section{BIBLIOGRAFÍA}

BERENGUER CONTRÍ, Gloria, CERVER ROMERO, Elvira, DE LA TORRE FERNÁNDEZ, Amparo y TORCAL TOMÁS, V. Ramón. El estilo directivo de las mujeres y su influencia sobre la gestión del equipo de trabajo en las cooperativas valencianas. CIRIEC-España, Revista de Economía Pública, Social y Cooperativa, $\mathrm{n}^{\mathrm{o}}$ 50, noviembre, 2004, p. 123-149.

BØHREN, Øyvind y STRØM, R. Øystein. Aligned, informed and decisive: Characteristics of value-creating Boards. Working paper, Norwegian School of Management, 2006.

BONN, Ingrid. Board Structure and Firm Performance: Evidence from Australia. Journal of Management \& Organization, 10, n ${ }^{\circ}$ 1, 2004, p. 14-24.

BROWN, A. H. David, BROWN, L. Debra y ANASTASOPOULOS, Vanessa. Women on Boards: Not Just the Right Thing . . . But the "Bright" Thing, The Conference Board of Canada, Report May, 2002. 
CAMPBELL, Kevin y MÍNGUEZ-VERA, Antonio. Gender Diversity in the Boardroom and Firm Financial Performance. Journal of Business Ethics, 83, nº 3, 2008, p. 435-451.

CARTER, David A., D'SOUZA, Frank, SIMKINS, Betty J. y SIMPSON, W. Gary. The gender and ethnic diversity of US boards and board committees and firm financial performance. Corporate Governance: An International Review, vol. 18 n 5, 2010, p. 396414.

CARTER, David A.., SIMKINS, Betty J. y SIMPSON, W. Gary. Corporate Governance, Board Diversity, and Firm Value. The Financial Review, no 38, 2003, p. 33-53.

CHAVES ÁVILA, Rafael; RIBAS BONET, María Antonia y SAJARDO MORENO, Antonia. Cumplimiento del primer principio: análisis de la discriminación por género en las cooperativas españolas. Revista de la Cooperación Internacional, ${ }^{\circ}$ 1, 2005, p. 101118.

ELÍO CEMBORAIN, Eunate. Techo de cristal y escalera de cristal en las cooperativas de Mondragón. Participación de las mujeres en los órganos de gobierno de las cooperativas. First International CIRIEC Research Conference on the Social Economy, Strengthening and Building Communities: The Social Economy in a Changing World, October 22-25, 2007 en Victoria, BC, Canada.

ERHARDT, Niclas L., WERBEL, James D. y Charles B. SHRADER. Board of Director Diversity and Firm Financial Performance. Corporate Governance: An International Review, April, 11, n² 2, 2003, p. 102-111.

ESTEBAN SALVADOR, María Luisa, GARGAllo CASTEL, Ana y PÉREZ SANZ, Francisco Javier. Composición del consejo rector y género en las cooperativas turolenses. REVESCO, Revista de Estudios Cooperativos, $\mathrm{n}^{\circ}$ 101, 2010, p. 7-27.

FARRELL, Kathleen A. y HERSCH, Philip L. Additions to corporate boards: the effect of gender. Journal of Corporate Finance, n ${ }^{\circ}$ 11, 2005 p. 85-106.

FEVECTA (1999). Las mujeres directivas en las cooperativas de FVECTA. Cuadernos Mujer y Cooperativismo, $\mathrm{n}^{\circ} .1$, Noviembre 1999 , http://www.coceta.coop/DptoSectoriales/Cuadernos/Primero/primero/OrgaCoopera/MUJE DIREC.htm, 28/02/2012.

FREGIDOU MALAMA, María. ¿Es necesario equilibrar el poder en las cooperativas entre mujeres y hombres?. CIRIEC-España, Revista de Economía Pública, Social y Cooperativa, $\mathrm{n}^{\circ}$ 50, 2004, p. 151-163. 
GARCÍA LARA, Juan Manuel, GARCÍA OSMA, Beatriz y MORA, Araceli. Gender diversity on the board and earnings quality. XIV Congreso AECA, Valencia 19-21 Septiembre, 2007.

GARCÍA JIMÉNEZ, Manuel. La necesaria armonización internacional del derecho cooperativo: el Caso español. REVESCO. Revista de Estudios Cooperativos, $n^{\circ}$. 102, 2010, p. 79-108.

MARTÍNEZ LEÓN, Inocencia María, ARCAS LARIO, Narciso y GARCÍA HERNÁNDEZ, Margarita. La influencia del género sobre la responsabilidad social empresarial en las entidades de economía social. REVESCO. Revista de Estudios Cooperativos, $\mathrm{n}^{\circ}$ 105, MONOGRÁFICO: La economía social y la igualdad de género, 2011, p. 143-172.

MATEOS DE CABO, Ruth, GIMENO NOGUÉS, Ricardo y ESCOT MANGAS, Lorenzo. Discriminación en los consejos españoles: análisis e implicaciones económicas. Revista de Economía Aplicada, nº 53, vol. XVIII, 2010, p. 131-162.

MATEOS DE CABO, Ruth, ITURROIZ DEL CAMPO, Javier y GIMENO NOGUÉS, Ricardo. La participación financiera y el papel de la mujer en la toma de decisiones de las sociedades cooperativas: los consejos de administración. Revista Europea de Dirección y Economía de la Empresa, vol. 18, nº. 3, 2009, p. 65-82

MATEOS DE CABO, Ruth; ESCOT MANGAS, Lorenzo y GIMENO NOGUÉS, Ricardo. Análisis de la presencia de la mujer en los Consejos de Administración de las mil mayores empresas españolas. Fundación de las Cajas de Ahorros. Documento de trabajo $\mathrm{n}^{\mathrm{o}} 263$, 2006.

MCPHAIL, Ken. Board Diversity, the Logic of Difference the Logic of Equivalence: A Critical Study of the Emergence of Corporate Democracy. Spanish Accounting Review, vol. $13, \mathrm{n}^{\circ} 1,2010$, p. 125-144.

ORELLANA ZAMBRANO, Waldo E.; CELIS EKSTRAND, Amanda. Género y capacidades directivas en cooperativas de trabajo asociado. Estudio exploratorio en ctas valencianas. REVESCO. Revista de Estudios Cooperativos, núm. 105, 2011, pp. 13-33.

PELLED, Lisa Hope, EISENHARDT, Kathleen M. y XING, Katherine, R. Exploring the Black Box: An Analysis of Work Group Diversity, Conflict, and Performance. Administrative Science Quarterly, no 44, 1999, pp. 1-28.

REDONDO CRISTÓBAL, Mercedes y JIMENO DE LA MAZA, Francisco Javier. Perfil económico-financiero de la empresa y perspectiva de género en el gobierno corporativo. XIII Congreso AECA, Oviedo, 22 a 24 de septiembre de 2005. 
RIBAS BONET, María Antonia. Mujeres y cooperativismo en la Comunidad Autónoma del País Vasco. Revista vasca de economía social. Gizarte ekonomiaren euskal aldizkaria, $\mathrm{n}^{\circ}$. 2, 2006, p. 153-175.

RIBAS, Ma Antonia y SAJARDO, María Antonia. La desigual participación de hombres y mujeres en la economía social: teorías explicativas. CIRIEC-España, Revista de Economía Pública, Social y Cooperativa, nº. 50, noviembre, 2005, pp. 77-103.

SAJARDO MORENO, Antonia; RIBAS BONET, M ${ }^{\mathrm{a}}$ Antonia; BENAVENT SANCHO, Matilde; SAZ GIL, Isabel; SERRA YOLDI, Inmaculada y VILAR FIGUEIRIDO, Montserrat. La Responsabilidad Social Interna de las empresas en España. Valencia: Tirant lo Blanch, 2009. 263 p. ISBN: 9788498767254.

SENENT VIDAL M ${ }^{\text {a }}$ José. ¿Cómo pueden aprovechar las cooperativas el talento de las mujeres? Responsabilidad social empresarial e igualdad real. Revista de Estudios Cooperativos, $\mathrm{n}^{\circ}$ 105, MONOGRÁFICO: La economía social y la igualdad de género, 2011, p.57-84.

SHRADER, Charles B., BLACKBURN, Virginia B. y ILES, Paul. Women in management and firm financial performance: an exploratory study. Journal of Managerial Issues, vol. 9, n³, 1997, p. 355-372.

SMITH, Nina, SMITH, Valdemar y VERNER Mette. Do Women in Top Management Affect Firm Performance? A Panel Study of 2500 Danish Firms. International Journal of Productivity and Performance Management, $\mathrm{n}^{\circ}$. 55, 2006, p. 569-593.

THE BOSTON CLUB. The 2004 Census of Women Directors and Executive Officers of Massachusetts Public Companies. The Boston Club, 2004. 